\title{
Capacidad Productiva de los pequeños productores del centro y sudoeste chaqueño. Su vulnerabilidad económica
}

\author{
Prof. Viviana C. Pertile
}

\section{Introducción}

Las grandes desigualdades sociales entre los países desarrollados y los países en vías de desarrollo son el fruto del actual modelo económico y productivo, triunfante en el mundo, basado en una constante necesidad de crecimiento cuantitativo y cuyo resultado es la acumulación del capital y la concentración de las riquezas de las Empresas Transnacionalesi', verdaderas dueñas del mundo.

En este mercado globalizado las reglas son: producir mucho y consumir otro tanto, como única forma de mantener en pie el sistema. Lógicamente, en este esquema del llamado "máximo crecimiento" se dejan de lado las consecuencias internas (inestabilidades económicas, sociales y políticas) y externas (agotamiento de los recursos naturales y humanos y el impacto sobre el medio ambiente).

Para el caso de Argentina, quien asumió en forma abierta un modelo neoliberal, dejó al mercado y a la apertura comercial como agentes reguladores del crecimiento, lo que en los hechos profundizó la incorporación de nuestro país a la "vorágine" de la globalización de la economía y a sus efectos, haciendo más dependiente y vulnerable nuestro tejido productivo, especialmente el agrario, que se encuentra en desventajas con el del contexto internacional más adelantado. Con ello han aumentado los problemas, multiplicándose la crisis agraria y de alimentación; se han agudizado la marginación, la competencia entre desiguales, la pobreza rural y urbana, los problemas medioambientales, la migración, y el agravamiento de las condiciones de vida, etc. Dentro de este contexto, los agricultores pierden capacidad de desarrollar sus propias opciones y son absorbidos por las innovaciones tecnológicas y los juegos financieros y de mercado de las empresas transnacionales. Por otra parte, dentro de este modelo internacionalizado, los pequeños y medianos productores son perjudicados por las mega-empresas, cuyo esquema de producción se caracteriza por el alto aporte de capital y tecnologíai".

Desde el punto de vista de la agricultura global, los pequeños agricultores de nuestra región no han podido asumir esta visión e incorporar la idea de una agricultura moderna y eficiente como la mejor alternativa para comenzar a solucionar los problemas nacionales, inclusive los del sector urbano industrial. Los agricultores han estado cautivos de ineficiencias crónicas en adquisición y utilización de insumos y equipos, administración de sus predios, conservación y almacenaje de sus cosechas y comercialización de lo producido. Fueron precisamente estas ineficiencias las que determinaron que la mayoría de los pequeños agricultores generaran una producción de volúmenes reducidos, de mala calidad, con costos unitarios de obtención muy altos y los vendieran a bajos precios. Esta falta de rentabilidad determinó la migración rural, propia de los últimos 20 o 30 años, por la expulsión de los agricultores hacia las periferias urbanas. Sus hijos y nietos en la mayoría de los casos son desempleados, cayendo en muchas ocasiones, estimulados por el consumismo, en la tentación del vicio, la prostitución y la delincuencia. De este modo en lugar de poder aportar riquezas y servicios a la sociedad en el campo, constituyen una carga para las ciudades (Lacki, 1995).

Estos hechos sociales, en parte disimulados por las políticas estatales (Plan trabajar, Jefes de hogar, etc.) para evitar una explosión social, están siendo enfrentados por los propios trabajadores del campo que intentan con sus acciones y políticas contrarrestar esta situación mediante su organización tanto en empresas campesinas, como en cooperativas. Todo ello con la idea de defender sus intereses colectivos, rescatar para sí los procesos productivos que les corresponden y su espacio social, en un contexto cada vez más globalizado.

En el marco de la realidad y de las tendencias anteriormente reseñadas, los pequeños agricultores se encuentran atravesando una crisis profunda: precios no remunerativos, productores altamente endeudados y sin posibilidad de acceso al crédito, lo que se traduce en una resultante social y económica que influye fuertemente sobre el contexto global del Chaco. Esta situación determinó que un grupo importante de pequeños y medianos productores, en su mayoría aborígenes dedicados al cultivo del algodón, se instalaran con carpas, a modo de protesta, en la Plaza Central de la ciudad de Resistencia iii, pasando de este modo a engrosar la 
ola de reclamos y paros de los distintos gremios estatales de nuestra provincia. Si bien se trató de una protesta genuina, por cuanto se vieron azotados por las condiciones climáticas adversas, los bajos precios del textil y la falta de instrumentación del fondo compensador algodonero, no desconocemos versiones que daban cuenta del trasfondo político que empañaba dicha protesta.

El presente trabajo tiene como objetivo analizar cómo las nuevas pautas económicas en la actividad agropecuaria han provocado el deterioro de la capacidad productiva del pequeño productor del Centro y Sudoeste de la Provincia del Chaco a fines de los años noventa, en el marco de la vulnerabilidad económica de la economía en la Argentina. Esta vulnerabilidad, al decir de Gambina y otros, 2002, tiene su eje central en el sector externo, y más particularmente en los condicionantes de la apertura externa que surgen desde la crisis de mediados de los ' 70 . El análisis de la situación socio productiva de los pequeños productores se realizó a partir de la utilización de distintas fuentes de información, como fueron el Censo Nacional de Población de 1991; datos del Ministerio de la Producción de la provincia del Chaco y básicamente las encuestas aplicadas a los pequeños productores. EPPACh-02 (Encuesta a Pequeños Productores Algodoneros del Chaco - 2001). Para establecer la muestra, se determinó en primer lugar el universo de estudio, constituido por 701 productores de hasta 100 ha de los departamentos Independencia (415) y General Belgrano (286). Estos datos fueron obtenidos de la Dirección de Catastro de la Provincia del Chaco quien proporcionó la información correspondiente al año 1995. Los mismos pertenecen al Padrón Valuatorio de Predios Rurales, cuya información sólo contiene a los propietarios y es utilizada para efectuar recaudaciones impositivas. Esto indica que la caracterización socioeconómica que realizamos se refiere exclusivamente a parcelas que se encuentra bajo el régimen de propiedad privada en cuanto al tipo de tenencia de la tierra. En segundo lugar se procedió a establecer la muestra resultante, a partir del uso del programa informático SAS y de cálculos estadísticos, quedando constituida por 65 productores -40 y 25 respectivamente- que representan el $9.2 \%$ del total de ambos departamentos, permitiéndonos un margen de error del $14 \%$. El tercer paso consistió en seleccionar los productores titulares (65) y los suplentes (40) mediante el uso de una tabla 1.5.1 de Selección al Azar (Snedecor, 1965). Por último, apoyándonos en las Cartas Catastral 1:100.000 de la Dirección de Catastro de la Provincia del Chaco, ubicamos cada una de las parcelas correspondiente a los 65 productores en la Carta Imagen Satelital 1:250.000, del Instituto Geográfico Militar.

\section{La Ocupación del espacio}

La actual distribución y tenencia de la tierra en nuestro espacio objeto de estudio, es el resultado de un proceso histórico, cuya valoración de las tierras y ocupación efectiva constituye un hecho relativamente reciente, ocurrido a finales del siglo XIX y principios del siglo XX.

El poblamiento sistemático del Chaco se inicia en el año 1870 cuando la consolidación de los poderes públicos nacionales permitió a los mismos concretar una labor de fomento. La organización político-administrativa, el arraigo de los inmigrantes, el establecimiento de las fuerzas militares y la distribución de la tierra fueron las medidas más importantes que crearon las condiciones adecuadas para la ocupación del territorio.

En las últimas décadas del siglo XIX se instalaron las primeras colonias agrícolas en el Oriente del Territorio chaqueño; pero el surgimiento de estos centros estuvo acompañado por la enajenación de grandes extensiones, por parte del gobierno. Este hecho, determinó la formación de grandes latifundios que abarcaron la mayor parte de las áreas factibles de ser ocupadas en esos años. A partir del año 1902 aproximadamente, con el avance hacia el interior, se crean numerosas colonias agrícolas y pastoriles, abandonándose el sistema de adjudicar tierras fuera de ellas.

Las primeras colonias agrícolas tuvieron un desenvolvimiento precario, debido fundamentalmente a la falta de incentivos para la actividad agrícola que se quería implantar y a las insuficientes comunicaciones. Otros de los factores adversos fueron el desconocimiento de los terrenos, las deficiencias en las mensuras y la mala administración. Estas falencias fueron más notorias en las concesiones otorgadas por el gobierno. En cambio, la situación fue diferente en la ocupación de las tierras fiscales del interior iniciada en 1907, donde pronto adquirieron una dinámica propia. Los factores decisivos fueron el tendido del ferrocarril ${ }^{\text {iv }}$-eje civilizador y progresista, el avance de la frontera militar y la valorización de los cultivos. Las colonias instaladas en estos años pronto se poblaron y al mismo tiempo se generalizaron las ocupaciones espontáneas en áreas no subdivididas (Shaller, 1986).

Nuestro espacio de estudio, se ubica en las colonias agrícolas $N^{\circ} 62$ denominada Colonia Agrícola General Necochea en el departamento Gral. Belgrano y la No 59 Colonia Agrícola 
Pampa Oculta en el departamento Independencia (Instituto de Geografía, 1989). Este último, tiene como cabecera a la localidad de Campo Largo, creada el 11 de julio de 1921 por Decreto del Poder Ejecutivo Nacional, pero el asentamiento de familias se remonta al año 1912 cuando se realiza la construcción de la línea del Ferrocarril Central Norte Argentino -hoy General Belgrano-. En el año 1918 llegaron desde la provincia de Corrientes los primeros colonos que se afincarían en estas tierras con sus respectivas familias, pero un año antes ya estaba establecido Don Jorge Traglio considerado uno de los primeros pobladores de Campo Largo ${ }^{v}$.

El departamento Gral. Belgrano, cuya cabecera es Corzuelai ${ }^{\mathrm{vi}}$, también se crea por decreto del Superior Gobierno de la Nación el 11 de julio de 1921. Sin embargo, el pueblo fue creado el 30 de abril de 1917; si bien los señores Cantón y Zappa fueron los dueños de estas tierras, el primer colonizador fue el Señor Baltazar Benito. En el año 1923 cuando tan sólo existía la estación del ferrocarril, llegaron otros pobladores: Juan Zarza, Aquilino de Santelésforo, Francisco Dabove, de nacionalidad española, quienes se dedicaron a la siembra de maíz y algodón, antes de la mensura de esas tierras (Ministerio de Educación, Cultura, Ciencia y Tecnología del Chaco, 1999).

\section{Capacidad productiva.}

\section{Variables e indicadores}

Los países de América Latina están enfrentando en la actualidad una profunda contradicción. Por un lado tienen una urgente necesidad de modernizar su agricultura, como única vía realista para lograr que ella sea eficiente, rentable y competitiva; y por otro lado, los gobiernos están disminuyendo el aporte de subsidios y créditos ( $y$ por ende de insumos y equipos) que tradicionalmente han sido propuestos para tecnificar y modernizar la agricultura.

Ante esta abrupta retracción del Estado, los agricultores inexorablemente tendrán que viabilizarse técnica y económicamente, a través de una mayor eficiencia y capacidad productiva, gerencial y organizativa con el agravante de que ésta deberá lograrse con menos créditos, insumos y equipos modernos; además, la agricultura rentable tendrá que ser sinónimo y consecuencia de agricultura eficiente, debido a que ya no existen subsidios para compensar ineficiencias productivas y gerenciales; sobrevivirán aquellos que se profesionalicen y que tengan actitudes y procedimientos empresariales como requisitos indispensables para lograr su rentabilidad y competitividad. (Lacki, 1995).

Las variables utilizadas para analizar la capacidad productiva del pequeño agricultor del Centro y Sudoeste Chaqueño fueron: superficie total, tenencia de la tierra, uso del suelo, producción agrícola-ganadera, mano de obra y nivel tecnológico.

Partimos de las variables tamaño y tenencia de la tierra, donde si bien nuestro objeto de estudio se centra en las pequeñas explotaciones cuya extensión no superan las 100 hectáreas, nos encontramos con predios cuya extensión no alcanza para la subsistencia familiar, es el caso de las parcelas agrícolas de menos de 5 ha cultivadas.

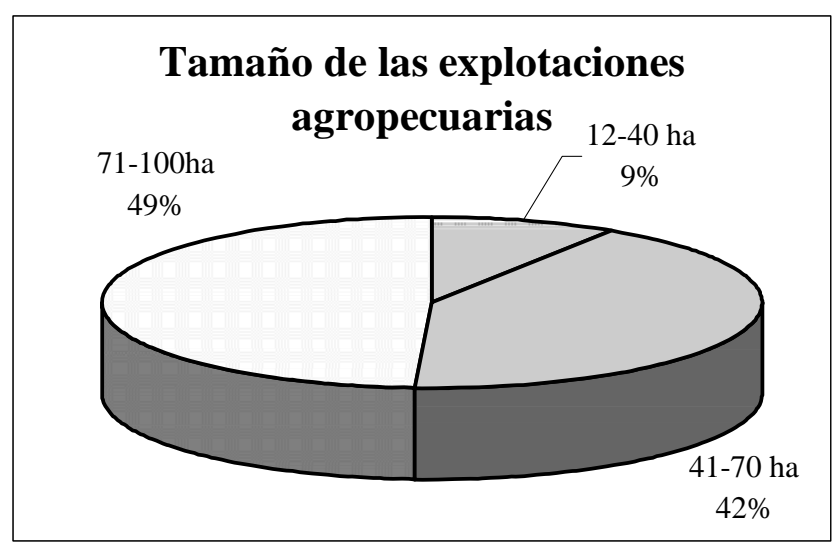

Fuente: EPPACh-01. $\mathrm{N}=65$

La extensión media de la superficie total de los 65 productores, es de es de 70 hectáreas, los predios más pequeños oscilan en las 12 y 15 hectáreas, y los más grandes alcanzan las 100 hectáreas. Para sintetizar el tamaño de las explotaciones agrupamos los predios en tres grupos para ver la proporción porcentual de cada uno de ellos respecto del total de la muestra (12 a 40 ha; 41 a 70 ha y de 71 a 100ha), tal cual lo muestra el gráfico.

Otra variable que nos interesó analizar fue el uso del suelo, relacionándolo con la producción, 
a los efectos de detectar fundamentalmente la actividad más importante en el establecimiento, representada por ejemplo por la cantidad de hectáreas cultivadas con los principales cultivos, si se practica la ganadería, la cantidad de cabezas y el destino de la misma, etc..

En primer lugar debemos mencionar la superficie total de cada uno de los usos del suelo en la muestra analizada. El resultado ha sido el siguiente: la superficie dedicada a la agricultura abarcó una extensión de 2359 hectáreas, la ganadera fue de 462 hectáreas y la superficie de monte nativo, sin explotar 1853 hectáreas. La proporción porcentual de estos usos del suelo son los siguientes:

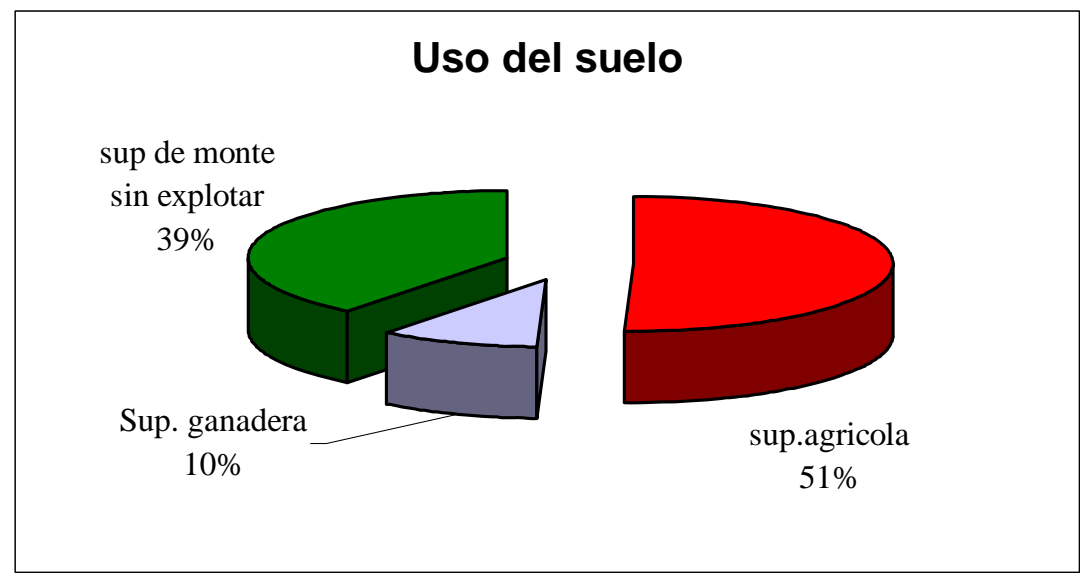

Fuente: EPPACh-01. $\mathrm{N}=$ 65

La información recabada nos permitió obtener la superficie media de los distintos usos del suelo de cada uno de los predios, predominando la media dedicada a la agricultura.

Fuente: EPPACh-01. $\mathrm{N}=65$

\begin{tabular}{|l|r|}
\hline \multicolumn{2}{|l|}{ Superficie media de Usos del suelo en hectáreas } \\
\hline Sup. media dedicada a la agricultura & 35,2 ha \\
\hline Sup. media dedicada a la ganadería & 7,1 ha \\
\hline Sup. media de monte & 28,6 ha \\
\hline Sup. media de monte en explotación & 0,3 ha \\
\hline
\end{tabular}

Teniendo la superficie dedicada a la agricultura de todos los productores, se obtuvo que el $64,6 \%$ de las hectáreas cultivadas se desarrolla en predios que oscilan entre las 3 y las 40 hectáreas; el $26,2 \%$ en campos de entre 41 y 60 ha y el 9,2\% en superficies de entre 61 y 90 hectáreas.

Otro de los aspectos que estudiamos en este punto fue el régimen jurídico, nos encontramos con que no existen variaciones, por cuanto el cien por ciento de los predios están bajo el régimen de propiedad ${ }^{\text {vii }}$.

\section{Producción agrícola-ganadera Principales cultivos}

Los productores agrícolas de la provincia del Chaco se han dedicado por muchos años al monocultivo del algodón, pero a partir de los años sesenta comienza la diversificación agrícola. En la provincia y en nuestra zona de estudio, se desarrollan una serie de cultivos, de los cuales siete u ocho son denominados "principales cultivos" -algodón, girasol, maíz, arroz, soja, sorgo granífero y trigo viii. De éstos, cinco se desarrollan en las distintas parcelas encuestadas y es el cultivo de algodón el que mayor superficie ocupó respecto del total de los principales cultivos, le siguió la soja, el maíz, el girasol y por último el sorgo granífero.

Con relación a ambas campañas analizadas ${ }^{i x}$, se pudo observar que, a excepción del algodón y el sorgo, en la campaña 1997/98 las superficies sembradas superaron a las de la campaña 1995/96. Los datos correspondientes a las hectáreas $(1.600,1.500$, etc.) representan la 
sumatoria de todas las hectáreas sembradas en los predios encuestados

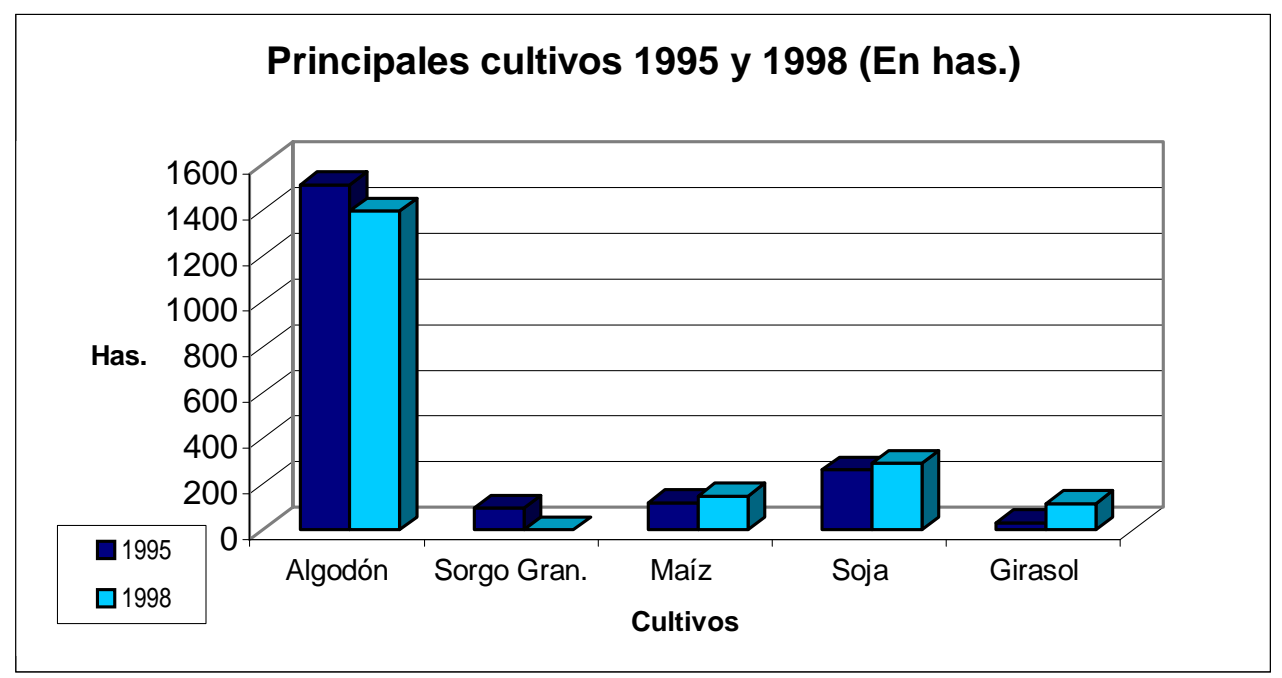

Fuente: EPPACh-01. $\mathrm{N}=65$

Para el caso del algodón, en la campaña 1995/96 se lo sembró en 58 explotaciones de las 65, las cuales sumaron 1510 hectáreas (esta superficie sembrada representó el 74,9\% del total cultivado); en tanto que en la campaña 1998/99 el número de predios disminuyó (55) al igual que la superficie total. El segundo cultivo en importancia, aunque con muchas hectáreas menos, fue la soja, que alcanzó en la campaña 1995/96 las 262 ha, cultivadas en solamente 6 explotaciones, alcanzando el $13 \%$ de la superficie total; repuntando en la campaña 1998/99 con 292 ha en 8 establecimientos. Mucho menos importante fueron las superficies sembradas en los restantes cultivos, el sorgo granífero representó el 4,7 \% de la superficie total, el maíz el $5,9 \%$ y el girasol el 1,9\%; este último tuvo un significativo repunte, de las 31 ha sembradas en la campaña 1995/96 paso a 116 ha en 1998/99 (del 1,9 al 5,9\% de la superficie total).

Estas variaciones observadas entre ambas campañas, referida a los cambios en las participaciones porcentuales de los cultivos, no escapa de las generales de la producción en la zona (Centro u Suroeste de la provincia del Chaco), por cuanto en los últimos años cultivos como la soja, el girasol, el sorgo granífero, permitieron la diversificación de la actividad en nuestra región.

\begin{tabular}{|l|c|c|c|c|}
\hline Cultivos & $\begin{array}{c}\mathbf{1 9 9 5} \text { ha } \\
\text { sembradas }\end{array}$ & $\begin{array}{c}\text { Cant. de } \\
\text { predios }\end{array}$ & $\begin{array}{c}1998 \text { ha } \\
\text { sembradas }\end{array}$ & $\begin{array}{c}\text { Cant. de } \\
\text { predios }\end{array}$ \\
\hline Algodón & $\mathbf{1 5 1 0}$ & $\mathbf{5 8}$ & $\mathbf{1 3 9 6}$ & $\mathbf{5 5}$ \\
\hline Sorgo Gran. & 95 & 1 & 0 & 0 \\
\hline Maíz & 119 & 12 & 148 & 15 \\
\hline Soja & $\mathbf{2 6 2}$ & $\mathbf{6}$ & $\mathbf{2 9 2}$ & $\mathbf{8}$ \\
\hline Girasol & 31 & 2 & 116 & 5 \\
\hline
\end{tabular}

Fuente: EPPACh-01. $\mathrm{N}=65$

Otro aspecto considerado en la agricultura, ha sido la comercialización de las cosechas, los resultados a los que arribamos han sido los siguientes: El 95,6 \% de los productores agrícolas entregan sus cosechas al acopiador, quien le entrega a cambio, dinero en efectivo; un pequeño porcentaje de productores entrega sus cosechas a cooperativas y desmotadoras $(4,4 \%)$. Si bien predomina el pago en efectivo, existe un pequeño porcentaje de productores $(1,5 \%)$ que suelen obtener como forma de pago, la entrega de mercaderías. Es posible observar en la zona de estudio, la modalidad de acopiadores/comerciantes, esto es cuando, el acopiador posee un comercio de ramos generales y es quien a los largo del año provee (a manera de 
crédito/cuenta corriente) de mercaderías a los colonos, estos son los que les entregan la producción a cambio de las cuentas. Generalmente, se trata de agricultores cuya superficie sembrada es muy escasa, por lo que su ganancia, muchas veces no alcanza para cubrir los gastos anuales y debe recurrir a otras fuentes de ingreso para hacer frente a su economía de subsistencia familiar.

Dentro de las actividades agropecuarias de los pequeños productores, nos pareció importante averiguar la cantidad de superficie dedicada a la horticultura, con vistas a aportar ingresos mediante la venta de sus productos, con gran asombro observamos que mas de la mitad de los colonos no tienen huertas, ni siquiera para su propio sustento; entre los que sembraban algunas hortalizas y verduras, predominaron las pequeñas superficies de un cuarto de hectárea, cuyo destino era únicamente el consumo familiar, predominando el cultivo de zapallos de distintas variedades.

\section{La ganadería}

La actividad agrícola, según se pudo apreciar es la actividad predominante, aunque no excluyente. En párrafos anteriores al hacer referencia a la superficie media de los distintos usos de suelo, observamos que la superficie dedicada a la ganadería es muy escasa, alcanzando una media de 7,1 hectáreas; esto es así como consecuencia de que se trata en términos generales de una ganadería de cría de tipo extensiva y destinada al autoconsumo y obtención de leche y sus derivados. Aquellos productores que tienen mayor número de cabezas de ganado vacuno, manifestaron destinarla a la comercialización, en cuyo caso la venta se realizaba a los vecinos de los pueblos próximos o en muy pocos casos a los carniceros de la zona. En el caso del ganado porcino y caprino, el destino es exclusivamente el consumo familiar; solamente uno de los encuestados tenia 8 cabezas de ganado equino, dijo destinarlos a la comercialización.

Del total de cabezas de ganado, el bovino es el que mayor proporción representó respecto del total de cabezas, con el $47.2 \%$, distribuido en 35 productores es decir el $53 \%$ del total; seguido por el porcino con un $19.9 \%$, que representan el $58 \%$ de los productores; en tercer lugar el caprino con $19.5 \%$, esto es el $26 \%$ de los colonos y finalmente equino con un $13.5 \%$ respecto del total, este ganado es el que mayor número de productores lo criaba y representaba el $63 \%$. Esto último se debía a que este ganado es utilizado como animal de tiro y representa la tracción animal utilizada para realizar la labor del agro.

A los efectos de ampliar el análisis, agrupamos los productores de acuerdo al número de cabezas de ganado vacuno -el de mayor proporción respecto del total- que tenían al momento de realizar la encuesta (año 2001). El resultado podemos apreciarlo en el siguiente gráfico, donde se advierte que la mayor proporción porcentual corresponde a los productores que no tienen ninguna cabeza de ganado bovino, el número de cabezas predominante está entre los 2 y 10 animales.

\section{Proporcion porcentual de ganado vacuno}

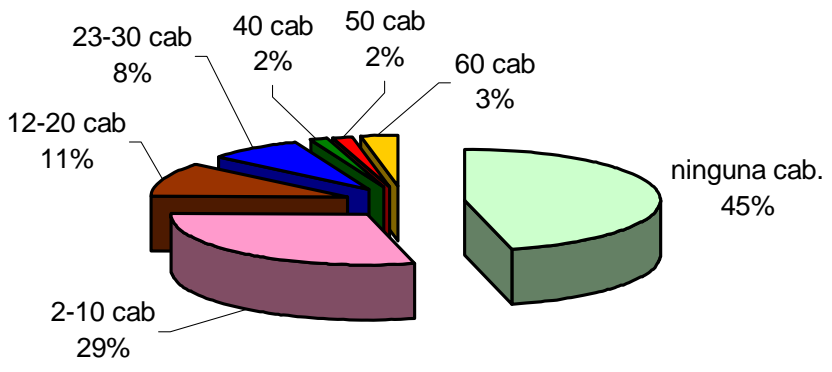

Fuente: EPPACh-01. $\mathrm{N}=65$

Para sintetizar podemos decir que la cantidad de animales y especies es reducida y variada (chivos, cerdos, vacas, caballos) observándose alto nivel de rusticidad como deficiencia en la 
alimentación, lo que da como resultado animales de bajo peso y calidad.

Otro de los usos del suelo, está representado por la cantidad de hectáreas ocupadas por el monte nativo, a pesar de lo irracional que fue la explotación forestal en estas tierras chaqueñas, pudimos ver a través de las encuestas que solamente el $19 \%$ de la superficie de monte está explotado. El mayor uso que se le da a la vegetación natural es el de la leña como combustible para cocinar.

La siguiente variable corresponde a la mano de obra, es decir, las personas que se encargan de realizar las actividades en los establecimientos. Utilizamos cinco indicadores, de los cuales dos fueron los más representativos. Uno era el productor con su familia y el otro, el personal denominado trabajador por tanto, que serían los cosecheros ${ }^{x}$. Como nuestra encuesta se centró en las pequeñas explotaciones, la mayor parte de las actividades, inclusive la cosecha, la realizan los integrantes del grupo familiar con muy pocos ayudantes para la cosecha (4 o 5); en el caso de establecimientos donde la superficie sembrada es mayor a 40 has se observó que se utilizaban mayor número de trabajadores por tanto (cosecheros).

Veamos tres cuadros estadísticos donde simplificamos lo explicado:

\begin{tabular}{|l|c|c|}
\hline $\begin{array}{c}\mathbf{N}^{\mathbf{0}} \text { de } \\
\text { cosecheros }\end{array}$ & $\begin{array}{c}\text { Proporción } \\
\text { porcentual }\end{array}$ & $\begin{array}{c}\text { Sup. dedicada } \\
\text { a la agricultura }\end{array}$ \\
\hline 15 cosech. & 1,5 & 40 ha \\
\hline 20 cosech. & 1,5 & 90 ha \\
\hline 30 cosech. & 1,5 & 60 ha \\
\hline 49 cosech. & 1,5 & 45 ha \\
\hline 50 cosech. & 1,5 & 49 ha \\
\hline
\end{tabular}

\begin{tabular}{|l|c|}
\hline $\begin{array}{c}\mathbf{N}^{\circ} \text { de } \\
\text { cosecheros }\end{array}$ & $\begin{array}{c}\text { Proporción } \\
\text { Porcentual }\end{array}$ \\
\hline ningún cosechero & 73,8 \\
\hline $1-10$ cosech. & 18,5 \\
\hline 15 cosech. & 1,5 \\
\hline 20 cosech. & 1,5 \\
\hline 30 cosech. & 1,5 \\
\hline 49 cosech. & 1,5 \\
\hline 50 cosech. & 1,5 \\
\hline
\end{tabular}

\begin{tabular}{|l|c|}
\hline $\begin{array}{c}\mathbf{N}^{\circ} \text { de personas en } \\
\text { mano de obra familiar }\end{array}$ & $\begin{array}{c}\text { Proporción } \\
\text { porcentual }\end{array}$ \\
\hline $1-4$ personas & 84,6 \\
\hline 5-9 personas & 12,3 \\
\hline
\end{tabular}

Fuente: EPPACh-01. $\mathrm{N}=65$

La siguiente variable utilizada para analizar la capacidad productiva fue el nivel tecnológico, es decir el tipo de equipamientos que posee el pequeño productor para realizar las labores agropecuarias.

Entre los primeros indicadores tenemos al tipo de tracción utilizada para realizar las actividades agrícolas, las encuestas nos indican que el $54 \%$ de los colonos utilizan la tracción animal, fundamentalmente el caballo, para realizar la preparación y siembra de la tierra, el $43 \%$ usa tractor propio y resto utiliza tractor en alquiler. Coincidentemente, 41 de ellos tenían caballos $(63 \%)$.

En relación a las características de los tractores, observamos que la mayoría de ellos son pequeños, de pocos caballos de fuerza y corresponden a modelos antiguos, los que nos habla a las claras de la escasa posibilidad que han tenido los colonos de cambiar su maquinaria. Del total de propietarios de tractores, 26 de los 65, el $85 \%$ correspondió a modelos de tractores que iban desde el año 1955 y 1980 y el 15\% restante entre 1983 y 1997 . Un $20 \%$ aproximadamente de los colonos suele en algunas campañas, recurrir al alquiler de tractores.

Entre los implementos agrícolas más usados por los colonos encontramos: arado, rastra, sembradora, cultivadora, mochila manual, entre las más comunes, de acuerdo la proporción de colonos que las poseían. El siguiente gráfico muestra la proporción de productores que poseían estos implementos/maquinarias agrícolas. 


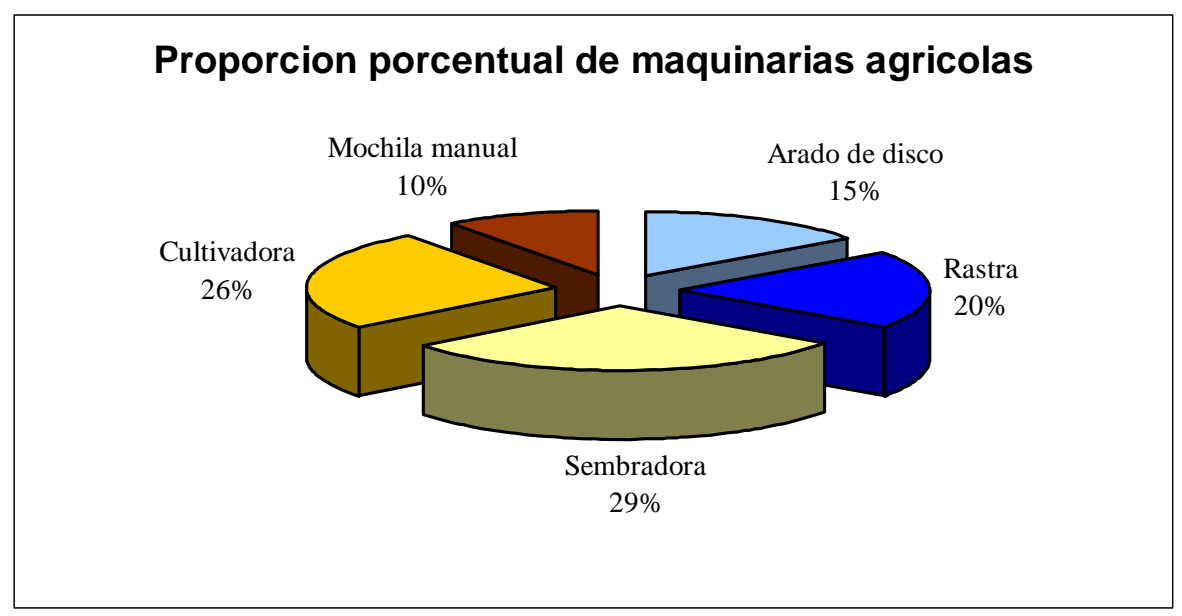

Fuente: EPPACh-01. $\mathrm{N}=65$

Otro indicador utilizado en el nivel tecnológico fue el uso de insumos y prácticas agrícolas e insumos y prácticas ganaderas, entre las primeras tenemos el uso de fertilizantes, agroquímicos, la práctica de la rotación, labranza cero, etc. y entre las segundas, el uso de antiparasitarios, pasturas cultivadas, suplementación, etc. Las prácticas más comunes en los pequeños productores fueron: la rotación y el uso de herbicidas, entre el 55 y el $65 \%$ y muy pocos el barbecho. Mas del $90 \%$ de los colonos no tenía conocimiento de la labranza cero y muy pocos manifestaron que habían escuchado hablar de ella. En relación a las prácticas ganaderas, los porcentajes en general fueron muy bajos, como consecuencia de la ganadería es una actividad prácticamente destinada al autoconsumo y el número de cabezas por productor, tal cual lo vimos en párrafos anteriores, es escaso.

También consideramos la variable equipamiento /(medio de transporte), es decir la presencia de algún tipo de movilidad ya sea para transportar los productos obtenidos de su producción agrícola/ganadera o para trasladarse a los pueblos más cercanos en busca de mercaderías e insumos para su predio. Asimismo la presencia de algún medio de transporte constituye un indicador de la calidad de vida, ya sea para acceder a algún centro asistencial en caso de enfermedad o simplemente de usarlo con fines recreativos. Los resultados fueron los siguientes: solamente $21 \%$ productores tenían camionetas, la mayoría de ellas no funcionaba por falta de dinero para mantenimiento y por tratarse de modelos de 25 a 30 años de antigüedad, solo dos de ellas pertenecían a modelos de la década del '80. Algo similar sucede con los camiones, únicamente en cuatro predios nos encontramos con ellos, de los cuales dos eran de la década del ' 50 y dos del ' 60 , todos sin funcionar. Otro tanto ocurría con los pocos automóviles con que nos encontramos, cinco, pero solamente tres en funcionamiento; finalmente el medio de transporte y de carga más usado era el carro, el $54 \%$ de los pequeños productores disponían de él, la antigüedad de estos variaron desde el año 1945 hasta los años'90.

Otra de las preguntas que incluimos en las encuestas para evaluar la marcha de las actividades agropecuarias en los últimos años fueron las inversiones; naturalmente que los resultados fueron muy desalentadores, solamente un productor compró unas pocas hectáreas, otro adquirió una mochila manual y 12 realizaron algunas mejoras, básicamente representadas por el alambrado de algunos sectores de sus predio, o la excavación de pozos de agua, o la reparación de corrales, entre los más comunes.

Para sintetizar las características de la capacidad productiva del pequeño productor, tenemos que, en primer lugar las explotaciones estudiadas corresponden a dos departamentos de Centro y Sudoeste chaqueño (Independencia y General Belgrano), abarcando únicamente los predios de hasta 100 hectáreas, donde el promedio se ubica en las70 hectáreas. Los datos relevados en la muestra indican el predominio absoluto de los propietarios (100\%). La actividad predominante es la agricultura, con un promedio de 32 hectáreas dedicadas a esta actividad.

La mano de obra predominante es de tipo familiar, los predios con mano de obra exclusivamente familiar alcanzan al $74 \%$ de la muestra. 
Los capitales con que cuentan los establecimientos son en general reducidos; las maquinarias, instalaciones y vehículos son antiguos, por dar el ejemplo del tractor, observamos que el $85 \%$ de los pocos tractores que existen pertenecen a modelos anteriores al año 1980. Otro tanto ocurre con el parque automotor, los pocos que encontramos no funcionan por falta de mantenimiento.

Con respecto a la tecnología, concluimos que las maquinarias son escasas, en solo uno de los predios se encontró que contaba con el equipamiento mínimo completo (arado, sembradora, cultivadora, mochila manual), el resto solo tenia uno o dos de ellos; por lo tanto para realizar la preparación y siembra de sus tierras recurren al préstamo o alquiler; asimismo pudo observarse que el parque de maquinarias era obsoleto. Lo mismo resultó con las prácticas e insumos tanto agrícolas como ganaderos, se practica lo elemental, creemos que esto se debe fundamentalmente a la falta de medios económicos pero también a la falta de información respecto a los nuevos insumos que existen en el mercado para obtener mejores rendimientos.

Con respecto al mercado, pudimos observar que no se trata en general de un mercado diversificado, ya que una alta proporción de productores $(95,6 \%)$ venden su producción agrícola al acopiador, en tanto que los que se dedican a criar algunos animales los vende a los vecinos de los pueblos próximos o en muy pocos casos a los carniceros de la zona.

Todas estas variables que acabamos de sintetizar, son las más representativas para evaluar la capacidad productiva del pequeño productor.

Utilizamos otras variables, que si bien lo dijimos anteriormente no se relacionan con la capacidad productiva, si intervienen en la caracterización general del pequeño agricultor. La edad y nivel de educación del productor son variables asociadas; la edad es una característica personal que incide en el comportamiento, incluyendo decisiones relacionadas con el uso de determinadas prácticas agrícolas, uso de tecnología, etc. La edad media de los productores era de 57 años, si bien no se trata de edad demasiado avanzada, las condiciones económicas son las que impiden realizar algún tipo de inversión o mejoras en los establecimientos El nivel educativo está asociado a la edad, en general los productores de mas de 40 años de edad y que pasaron su infancia y adolescencia en zonas rurales con limitaciones para el acceso a la educación, tienen bajo nivel de escolaridad, los datos que recogimos nos dieron como resultado que el $73 \%$ no completó la escuela primaria.

Otras variable fue la denominada fuente de ingreso, el ingreso total del campesino está compuesto por ingresos parciales, sin embargo existen una o dos fuentes principales de obtención de dinero, alrededor de las cuales se estructuran los demás ingresos. La entrada de dinero proveniente de la venta de la producción agrícola es lo más importante, nos encontramos con que el $85 \%$ de los colonos, tiene a la explotación de la tierra como la principal fuente de ingreso, combinado en muy pocos casos, con los de origen pecuario. Pero también encontramos que existen otras fuentes de ingreso que ayudan a paliar la situación por la que están atravesando, el $67 \%$ de las explotaciones tiene diferentes ayudas económicas, tales como jubilaciones, empleos relacionados con la administración pública como ser los maestros, changas ocasionales, ayudas familiares, peón, entre las más comunes. La migración de algunos integrantes del grupo familiar, en busca de trabajo fuera del campo, suele ser una estrategia para ayudar a engrosar los ingresos, pero en general no es muy significativo. En términos generales se puede decir que esta explotación tiene una base de corte agropecuario.

\section{Conclusión}

A partir de la información recabada, hemos podido observar que en los últimos años el sector agrícola local, regional y nacional atravesó y atraviesa profundos e intensos problemas económicos que potencian el grado de vulnerabilidad económica de los pequeños productores de nuestra provincia.

En relación a las características de la capacidad productiva del pequeño productor, señalamos en primer lugar, que las explotaciones estudiadas corresponden a dos departamentos de centro y sudoeste chaqueño (Independencia y General Belgrano), abarcando únicamente predios de hasta 100 hectáreas, con un promedio de 70 hectáreas. La actividad sobresaliente 
es la agricultura, con un valor medio de 32 hectáreas dedicadas a esta actividad. Con una mano de obra predominantemente de tipo familiar, alcanzando el $74 \%$ de los predios de la muestra.

El capital con que cuentan los establecimientos son en general muy reducidos; las maquinarias, instalaciones y vehículos son antiguos, por ejemplo, el $85 \%$ de los escasos tractores que existen pertenecen a modelos anteriores al año 1980. Otro tanto ocurre con el parque automotor, los pocos vehículos encontrados no funcionan por falta de mantenimiento.

Con respecto a la tecnología, concluimos que las maquinarias son escasas, en sólo uno de los predios se encontró que contaba con el equipamiento mínimo completo (arado, sembradora, cultivadora, mochila manual), el resto solo tenía uno o dos de ellos; por lo tanto para realizar la preparación y siembra de sus tierras recurren al préstamo o alquiler; asimismo pudo observarse que el parque de maquinarias era obsoleto. Lo mismo resultó con las prácticas e insumos tanto agrícolas como ganaderas; se practica lo elemental. Esto se debe fundamentalmente a la falta de medios económicos pero también a la escasa educación y a la falta de información respecto a nuevas técnicas y nuevos insumos que existen en la actualidad para obtener mejores rendimientos.

En relación al mercado, se observa que no es en general diversificado, ya que una alta proporción de productores (95,6 \%) venden su producción agrícola al acopiador, en tanto que los que se dedican a criar algunos animales los venden a los vecinos de los pueblos próximos o en muy pocos casos a carniceros de la zona.

En cuanto al ingreso familiar, se rescató que el ingreso total del campesino está compuesto por ingresos parciales, sin embargo existen una o dos fuentes principales de obtención de dinero, alrededor de las cuales se estructuran los demás ingresos. La entrada de dinero proveniente de la venta de la producción agrícola es la más importante; el 85\% de los colonos, tiene a su explotación como la principal fuente de ingreso, combinada en muy pocos casos, con los productos de origen pecuario. Pero también encontramos que existen otras fuentes que ayudan a sostener la situación en la familia; en el $67 \%$ de los casos relevados existen diferentes ayudas económicas, tales como jubilaciones, empleos relacionados con la administración pública como ser los maestros, changas ocasionales, ayudas familiares y trabajo a destajo como peón, entre las más comunes. La migración de algunos integrantes del grupo familiar, en busca de trabajo fuera del campo, suele ser una estrategia para ayudar a engrosar los ganancias, pero en general no mostró ser muy significativa.

En estas condiciones, nos encontramos con pequeños productores agropecuarios cuyo ingreso principal deriva de la venta de sus productos agrícolas, destinado a gastos vinculados con la misma producción y los que exigen la compra de alimentos básicos que no pueden ser cubiertos con la propia producción. Estos magros ingresos permiten calificar al pequeño productor como pobre rural, por cuanto necesita cada vez mayores esfuerzos para obtener el sustento diario. La falta de inversión, por parte de los gobiernos que se fueron sucediendo en el tiempo (mejoramiento de caminos, luz, agua potable, etc) trae aparejado niveles de vida poco dignos, donde las necesidades básicas no son cubiertas en su totalidad y con tendencia a incrementarse con el correr del tiempo.

A partir de la información recabada, hemos podido observar que el sentido impuesto por los programas de ajuste, lejos de reencauzar el sistema económico de modo progresivo, potenció los problemas del subdesarrollo, creando nuevas y mayores restricciones al crecimiento de los distintos sectores de la provincia --incluido el agro--, ampliando las condiciones de inestabilidad económica.

\section{Bibliografía}

ALEXANDRATOS, Nikos (1995): Agricultura mundial hacia el 2010. Estudio de la FAO. Madrid, España. Ed. Mundi-Prensa. 
ARANDA BAEZA, X (1981): Empleo, migración rural y estructura productiva agrícola. Santiago de Chile. FLACSO.

ARNAUD, Vicente G. (1999): MERCOSUR. Unión Europea, Nafta, y los procesos de Integración Regional. Buenos Aires. ABELEDO -PERROT.

BARBEITO, A y LO VUOLO, R (1995): La Modernización Excluyente. Transformación Económica y Estado de Bienestar en Argentina. Buenos Aires, Losada S.A.

BESIL, A. y PIEDRA, D. (2000): "Indicadores sociales relevantes de la región algodonera argentina" En PROINTAL, Programa Integral Algodonero, Capítulo VII. Resistencia, Chaco. Gobierno de la Provincia del Chaco, INTA., UNNE,

BIDASECA, K; GRAS, C. y MARIOTTI, D.(2000): "Las transformaciones del mercado de trabajo en el escenario rural: viejos y nuevos procesos de negociación". En: III Congreso Latinoamericano de Sociología del Trabajo. Buenos Aires. Asociación Latinoamericana de Sociología del Trabajo.

BOSERUP, Ester (1984): Población y cambio tecnológico. Mendoza. EDIUNC.

BROWN, Harres B. y WARE, Jacob O(1961): Algodón. México, D.F. UTHEA.

BRUNIARD, Enrique D.(1978): El Gran Chaco Argentino. Ensayo de interpretación geográfica, GEOGRÁFICA 4, Resistencia, Chaco. Facultad de Humanidades, UNNE.

BRUNIARD, Enrique D.(1981): El Clima de las planicies del norte argentino. Facultad de Humanidades UNNE. Resistencia, Chaco.

CÁCERES, Javier (1998): "Globalización y reestructuración del sector agrícola", En II Congreso Técnico Empesarial, Rosario 27 y 28 de agosto de 1998, Federación Agraria Argentina y Programa Fortalecer.

CARDOZIER, U. R (1962): Cultivo y producción del Algodón. México, D.F. Herrero.

CARLEVARI, Isidro J. F (1996): La Argentina: Estructura Humana y Económica. $2^{\circ}$ ed. Buenos Aires. Ed. Macchi,

CASTELLS, Manuel (1998). "Entender nuestro mundo", en Revista de Occidente, España. Fundación José Ortega y Gasset.

CEIL, Adela (1991): De la agricultura tradicional a la tecnológica. Madrid. Cincel.

CIMMYT, (1993): Programa de economía. La adopción de tecnologías agrícolas: guía para el diseño de encuestas. México. D.F.

DAVID, Jean (1971): Le Coton et L' Industrie Conniere. Paris. Presses Universiteries de France.

DEREWICKI, J: (1997), en Diario Norte, 9 de Abril de 1997. Resistencia, Chaco..

DIAZ ALVAREZ, José R. (1990): Geografía y agricultura: componentes de los espacios agrarios. Madrid, Cincel.

ESCOBAL Y PONCE (2000): Innovaciones en la lucha contra la pobreza rural en América Latina. Documento preparado para la CEPAL, para ser presentado en el Encuentro de Altos Directivos contra la Pobreza Rural (Santiago de Chile, 27 y 28 de enero del 2000) organizado por la CEPAL, FAO, el BID y RIMISP.

ESTEFANÍA, Joaquín (1996): La nueva economía. La globalización. Madrid, España. Ed. Debate SA.

FERRER, Aldo (1997): Hechos y ficciones de la globalización. Buenos Aires, Fondo de Cultura Económica, S.A. 
pequeños también pueden". En Serie Desarrollo Rural No 11. FAO. Organización de las Naciones Unidas para la Agricultura y la Alimentación.

GAMBINA, Julio, y otros, 2002: Vulnerabilidad externa y dependencia de la economía argentina. En Gambina, Julio Compilador, La Globalización económico-financiera Su impacto en América Latina. Colección Grupos de Trabajo de CLACSO. Consejo Latinoamericano de Ciencias Sociales / CLACSO. Compilador

GELMAN, Susana (1995): "Análisis del Producto Bruto Geográfico de la Provincia del Chaco. En: Indicadores Económicos. Resistencia, Chaco. Revista del Departamento de Finanzas Año 4, № 22. Facultad de Ciencias Económicas de la UNNE.

GIARRACA, Norma (2001): ¿Una nueva ruralidad en América Latina? Colección grupos de trabajos de desarrollo rural. CLACSO - ASIDI. Buenos Aires, Argentina.

GIDDENS, Anthony (1999): Un Mundo Desbocado. Los efectos de la globalización en nuestras vidas .Madrid. Ed. Taurus.

GIGET, Marc (1995), en GODET, Michel De la anticipación a la acción, Barcelona. Marcumbo editores.

GONZALEZ, Mirta (1988): "Economía agroganadera de vocación exportadora" En Roccatagliata, J. A. La Argentina: geografía general y los marcos regionales. Grupo Editorial Planeta Argentina. Buenos Aires, Argentina.

HERNÁNDEZ ARACENA, R y THOMAS WINTER, C (1999): "Educación, Modernidad y Desarrollo Rural" . En : Revista Enfoques Educacionales Vol.2 №1 1999.

INSTITUTO DE GEOGRAFÍA de la Facultad de Humanidades de la UNNE (1987). Atlas Geográfico de la Provincia Chaco, Tomo I: El Medio Natural. "Geográfica 5" Resistencia, Chaco.

INSTITUTO DE GEOGRAFÍA de la Facultad de Humanidades de la UNNE (1989). Atlas Geográfico de la Provincia Chaco, Tomo II: La tierra y la Agricultura. "Geográfica 6". Resistencia, Chaco.

INSTITUTO DE GEOGRAFÍA de la Facultad de Humanidades de la UNNE (1990). Atlas Geográfico de la Provincia Chaco, Tomo III: La Población y el Hábitat "Geográfica 7". Resistencia, Chaco.

INSTITUTO NACIONAL DE ESTADÍSTICAS Y CENSOS (1991): Censo Nacional de Población y Vivienda 1991, Serie B № 25, Total del País, INDEC, Bs,As.

INSTITUTO NACIONAL DE ESTADÍSTICAS Y CENSOS (1994): "Mapas de la Pobreza en la Argentina". Comité Ejecutivo para el Estudio de la Pobreza. Bs,As, Argentina.

INSTITUTO NACIONAL DE TECNOLOGÍA AGROPECUARIA (INTA) EERA de Presidencia Roque Sáenz Peña. Mapa de Capacidad de Uso del Suelo en el Chaco. Escala 1:500.000.

INTA (Instituto Nacional de Tecnología Agropecuaria) (1990): Atlas de suelos de la República Argentina, Tomo 1. Capacidad del uso del suelo, Buenos Aires.

INTA (Instituto Nacional de Tecnología Agropecuaria),UNNE (Universidad Nacional del Nordeste) y Gobierno e la Provincia del Chaco (2000): PROINTAL (Programa Integral Algodonero). Resistencia, Chaco.

KLIKSBERG, Bernardo (2000). "Replanteando el problema social: más allá de mitos y slogans", En Hintze, Susana -comp.- Estado y Sociedad, Las políticas sociales en los umbrales del siglo XXI, Buenos Aires. Eudeba.

KUGLER, Nicolás Diego (1994): Razones para migrar. Una estudio de población del Chaco Algodonero. Tesis de Licenciatura (Inédito). UBA. Buenos Aires.

LACKI, Polan, (1995): Buscando soluciones para la crisis del agro, ¿en la ventanilla del banco, 
o en el pupitre de la escuela?. Santiago de Chile.

LARGIÉRE, Robert (1968): El algodón. Técnicas agrícolas y producciones tropicales. Blume. Barcelona.

LARRAMENDY, Juan C.(1980): La Economía Algodonera Chaqueña y sus perspectivas. Resistencia, Chaco, Departamento de impresiones y diagramaciones, Cosecha.

LOPEZ PIACENTINI, Carlos Primo (1968): Evolución agrícola del Chaco, del reinado del algodón a la diversificación en el Chaco actual. Región. Resistencia,

MAEDER, Ernesto J.A (1967): Historia del Chaco y sus pueblos. 1862-1930. Buenos Aires, El Ateneo.

MANOILOFF, Raúl O (2001): El cultivo del algodón desde 1950 a la actualidad (la etapa de crisis) Resistencia, Chaco. Meana y Meana.

MITNIK, Oscar y MONTOYA, Silvia (1995): "Pobreza y distribución del ingreso. Dinámica y característica. Gran Buenos Aires, 1974-94", en Estudios, julio-setiembre.

MONETA, Carlos (1994): "El proceso de globalización: Percepciones y desarrollo". En Las Reglas del Juego. América Latina, globalización y regionalismo. Buenos Aires. Ed. Corregidos.

PALAU VILADESAU, Tomás y, F. Edson Teófilo (1992): "Qué ocurrirá con el campesinado? Reflexiones sobre exclusiones, participación y autogestión en la sociedad agraria paraguaya" En: BASE investigaciones sociales № 36 . Programa de Estudios agrarios y campesinos. Asunción, Paraguay.

PATRONI, Anibal (1999): "Líneas Básicas para un Programa de Desarrollo Rural Integral". Material suministrado en el cursado de la Maestría en Desarrollo Social, Resistencia-Chaco, 2000.

PAZ, R. (1997): "Pobreza rural y medio ambiente. Su análisis en un contexto globalizado". En Revista Realidad Económica No 152. Ed. IADE. Buenos Aires

PEPE, Norberto C. y DEREWICKI, José V. (1997): Su majestad el Algodón. Resistencia, Chaco, Meana y Meana.

PERONA, Nélida y Otros (2001): Vulnerabilidad y Exclusión Social. Una Propuesta metodológica para el estudio de las condiciones de vida de los hogares. Universidad de Bio Bio. Santiago.Chile. www.ubiobio.cl/cps/ponencia/doc)

PERTILE, Viviana (2003): Ampliación de la frontera agropecuaria chaqueña: El Oeste Chaqueño y el cultivo algodonero. IIGHI, Resistencia, 2003.

ROFFMAN, A. y MANZANAL, M. (1989). Las economías regionales de la Argentina. Crisis y políticas de desarrollo, Buenos Aires. Bibliotecas Universitarias - Centro Editor de América Latina,

ROFMAN. Alejandro B. (2000): Desarrollo Regional y Exclusión Social. Transformaciones y crisis en la Argentina contemporánea. Buenos Aires, Amorrortu editores.

SAXE-FERNÁNDEZ, John (1994): Nafta: Los cruces de la geopolítica y geoeconomía del capital. En El Mundo Actual: Situación y Alternativas. México. Centro de Investigaciones Interdisciplinarias en Humanidades.

SCHALLER, Enrique C (1986): "La Colonización en el Territorio Nacional del Chaco en el período 1969-1921" En Cuadernos de Geohistoria regional № 12, Resistencia, Chaco. Instituto de Investigaciones Geohistóricas, CONICET-FUNDANORT.

Ernest, Ed. Konrad-Adenauer-Stiftung. Buenos Aires, Argentina.

SNEDECOR, George (1965): Statical Methods. Applied to Experiments in Agriculture and Biology. Ames, lowa, USA. 
TAYLOR, Edward J. (2001): Migración: Nuevas dimensiones y características, causas y consecuencias e implicancias para la pobreza rural. Ed. Kostas G. Stamoulis. Roma Italia.

VALEIRO, Alejandro (1998): El Nuevo Algodón ¿Son sustentables los cambios recientes del sector algodonero Argentino? Publicación del INTA, Santiago del Estero. En página Web HIPERVÍNCULO httttp://WWW.INTA.com.ar"ww.inta.com.ar

YANES, Luis y LIBERALI, Ana María (1986): Aportes para el estudio del espacio socioeconómico. Buenos aires, Argentina. Ed. El Coloquio.

Fuentes

DIRECCIÓN DE CATASTRO DE LA PROVINCIA DEL CHACO: Padrón valuatorio de predios rurales, 1995. Resistencia Chaco.

DIRECCIÓN DE ESTADÍSTICAS Y CENSOS: Censo Nacional Agropecuario de 1988. № 17. Provincia del Chaco. Buenos Aires 1991.

DIRECCIÓN DE PLANIFICACIÓN, CAPACITACIÓN Y EVALUACIÓN del Ministerio de la Producción de la Provincia del Chaco. Serie Estadística № 16. Enero 1996.

INSTITUTO NACIONAL DE ESTADíSTICAS Y CENSOS: Censo Nacional de Población de 1991.

MINISTERIO DE ECONOMÍA OBRAS Y SERVICIOS PÚBLICOS. .Secretaría de Programación económica. Provincia del Chaco. El Chaco en cifras, Resistencia. Chaco, 1999

MINISTERIO DE EDUC, CULTURA, CIENCIA Y TECNOLOGÍA DE LA PROVINCIA DEL CHACO: Ciudades y pueblos de la Provincia del Chaco. Dirección General de Bibliotecas, Biblioteca Pública Leopoldo Herrera Resistencia, 1999.

MINISTERIO DE LA PRODUCCIÓN Provincia del Chaco. Compendio de la Producción, Resistencia. Chaco, 1999.

\section{Notas}

\footnotetext{
' Las Empresas Transnacionales gastan más que los países líderes en la investigación y desarrollo de tecnologías. El gasto combinado de las diez más grandes transnacionales de los Estados Unidos, por ejemplo sobrepasa el de los gobiernos de Francia y el Reino Unido. Este poder de la ETS es uno de los rasgos distintivos de la actual situación de la economía mundial., de la denominada "globalización de los mercados". (Paz, 1997)

ii Cf. Gaitán Arciniegas, Jorge y Lacki, Polan (1993).

iii La ocupación de referencia tuvo lugar en la Plaza 25 de mayo de 1810, de la ciudad de Resistencia, capital de la provincia del Chaco, durante los meses de junio y julio del año 2005.

v La construcción del ferrocarril se inicia desde Barranqueras en 1909 y llega hasta Avia Terai (Departamento Independencia) en 1914. Allí se produjo el empalme con la línea que se tendía desde Añatuya y Quimilí y atravesaba el Suroeste del Territorio. (Schaller, 1986)

${ }^{\vee}$ El origen del nombre es exótico sin significado y valor histórico, tomó este nombre por que allí podía observarse el Fortín Las Chuñas (Ministerio de Educación, Cultura, Ciencia y Tecnología del Chaco, 1999).

${ }^{v i}$ Corzuela debe su nombre a un cuadrúpedo rumiante muy ligero que habitaba en la zona y que pertenece a la familia de los guasunchos, cérvido del género Mazama, (Ministerio de Educación, Cultura, Ciencia y Tecnología del Chaco, 1999).

vii Cabe aclarar que la información para la elaboración de la muestra fue obtenida del Padrón Valuatorio de Predios Rurales, proporcionado por la Dirección de Catastro de la Provincia del Chaco, donde figuran únicamente los propietarios, por cuanto se trata de información utilizada para realizar las recaudaciones impositivas

viii La denominación de "principales cultivos" se realiza en función a la mayor superficie cultivada y producción, respecto del total de los cultivos que se desarrollan en la provincia del Chaco. (Ministerio de la Producción. Dirección de Planificación, del Ministerio de ganadería y agricultura de la provincia del Chaco, 1999).

${ }^{i x}$ Decidimos tomar estas dos campañas para apreciar si hubo diferencias entre ambas, por cuanto la campaña 1995/96 representó el récord histórico en relación a cantidad de hectáreas sembradas, en tanto que en el año 1998 la provincia del Chaco estuvo afectada por excesos hídricos, ocasionando graves daños al sector agropecuario En el caso de la ganadería, interesa la cantidad de cabezas por tipo de ganado y el destino del mismo
} 
${ }^{x}$ Los otros indicadores utilizados en las encuestas fueron: número de personas en mano de obra familiar asalariada; número de personas contratadas (solamente se dio en dos establecimientos con 1 y 2 personas) y número de jornales pagados en forma transitoria (este tipo se dio en cuatro establecimiento y solamente se pagaron a 1 o 2 jornales en cada uno de ellos), este último tipo corresponde por ejemplo a los jornales pagados para realizar tareas como carpida, destronque, alambrado, entre las más comunes) 